\title{
Pretreatment Lung Immune Prognostic Index Is a Prognostic Marker of Chemotherapy and Epidermal Growth Factor Receptor Tyrosine Kinase Inhibitor
}

\author{
Seigo Minami ${ }^{\mathrm{a}}$, , Shouichi Ihara ${ }^{\mathrm{a}}$, Kiyoshi Komuta ${ }^{\mathrm{a}}$
}

\begin{abstract}
Background: Lung immune prognostic index (LIPI) was recently developed on the basis of the combination of baseline derived neutrophil to lymphocyte ratio (dNLR) and lactate dehydrogenase (LDH). This index was demonstrated as a specific biomarker of immune checkpoint inhibitors for non-small cell lung cancer (NSCLC). We aimed to show that LIPI may be a useful biomarker of cytotoxic chemotherapy and epidermal growth factor receptor (EGFR) tyrosine kinase inhibitor (TKI) for NSCLC.
\end{abstract}

Methods: We retrospectively collected 175 wild-type EGFR adenocarcinomas, 131 NSCLCs harboring mutant EGFR and 110 squamous cell carcinomas. All patients initiated first-line cytotoxic chemotherapy or EGFR-TKI monotherapy between July 2007 and August 2017 at our hospital. These patients were divided into good, intermediate and poor LIPI groups. We compared their overall survival (OS) and progression-free survival (PFS). Multivariate analyses detected prognostic and predictive factors of OS and PFS.

Results: The good LIPI group survived longer than the intermediate and poor LIPI groups in wild-type EGFR adenocarcinoma (good, intermediate and poor LIPI groups: median 19.6, 11.5 and 3.3 months, $\mathrm{P}<0.01$, respectively) and mutant EGFR NSCLC (45.4, 25.6 and 15.7 months, $\mathrm{P}<0.01$ ). The PFS of good LIPI group was significantly longer that those of the other two groups in mutant EGFR NSCLC $(16.6,12.6$ and 8.3 months, $\mathrm{P}<0.01)$. The intermediate group (hazard ratio (HR) $1.49,95 \%$ confidential interval $(\mathrm{CI}) 1.03-2.15, \mathrm{P}=0.04$ ) of wild-type EGFR adenocarcinoma, intermediate (HR 2.30, 95\% CI $1.33-3.99, \mathrm{P}<0.01$ ) and poor (HR 2.76, 95\% CI $1.03-7.42, \mathrm{P}=$ 0.04) groups of mutant EGFR NSCLC were independent prognostic factors of poor OS. The intermediate (HR 1.57, 95\% CI $1.01-2.44$, $\mathrm{P}=0.04$ ) and poor (HR 2.63, 95\% CI $1.14-6.07, \mathrm{P}=0.02$ ) groups were significant prognostic factors of PFS of mutant EGFR NSCLC.

Conclusions: LIPI was an independent prognostic factor of chemo-

Manuscript submitted December 17, 2018, accepted December 27, 2018

aDepartment of Respiratory Medicine, Osaka Police Hospital, 10-31 Kitayama-cho, Tennoji-ku, Osaka 543-0035, Japan

${ }^{b}$ Corresponding Author: Seigo Minami, Department of Respiratory Medicine, Osaka Police Hospital, 10-31 Kitayama-cho, Tennoji-ku, Osaka 543-0035, Japan. Email: Seigominami@oph.gr.jp

doi: https://doi.org/10.14740/wjon1179 therapy for adenocarcinoma with wild-type EGFR and of EGFR-TKI for NSCLC harboring mutant EGFR. Thus, LIPI was not a specific biomarker for ICI therapy, but a useful biomarker for chemotherapy and EGFR-TKI therapy in specific subsets of NSCLC.

Keywords: Lung immune prognostic index; Derived neutrophil to lymphocyte ratio; Lactate dehydrogenase; Non-small cell lung cancer; Adenocarcinoma; Squamous cell carcinoma; Epidermal growth factor receptor tyrosine kinase inhibitor; First-line cytotoxic chemotherapy

\section{Introduction}

Non-small cell lung cancer (NSCLC) has been classified into several subsets according to histological and genetic characteristics in the past decade: squamous cell carcinoma and nonsquamous NSCLC with or without driver mutations such as epidermal growth factor receptor (EGFR) mutation and anaplastic lymphoma kinase (ALK) rearrangement. Nowadays, programmed death-ligand 1 (PD-L1) expression in tumor has become a new biomarker of immune checkpoint inhibitors (ICIs) for NSCLC. Treatment strategy is various among subsets. For patients with NSCLC harboring a driver mutation, a specific tyrosine kinase inhibitor (TKI) is recommended as the first-line regimen. For squamous cell carcinoma and NSCLC without any driver mutations, either conventional cytotoxic chemotherapy or ICI is considered as the first-line regimen according to PD-L1 expression in tumor. Conventional chemotherapeutic regimen is also different between squamous and non-squamous NSCLC.

Lung immune prognostic index (LIPI) was recently developed on the basis of the combination of baseline derived neutrophil to lymphocyte ratio (dNLR) greater than 3 and lactate dehydrogenase (LDH) greater than upper limit of normal (ULN) range of each center. This index stratifies patients into three groups (good, intermediate and poor) according to the number of factors. For patients with advanced NSCLC, poor baseline LIPI was correlated with poor outcomes of overall survival (OS), progression-free survival (PFS) and disease control rate (DCR) for ICIs therapy of PD-1/PD-L1 inhibitors, but not for chemotherapy [1]. Thus, LIPI has been expressed as a specific biomarker for ICIs. The dNLR consists of easily measured hematological components of leukocyte and neutro- 
phil counts, and has similar prognostic value to the neutrophil to lymphocyte ratio (NLR) [2]. Compared with similar inflammation-based scores, dNLR has an advantage of widespread utilization, but has not been evaluated in NSCLC. Both LDH [3-5] and NLR [6-8] have been demonstrated as a useful prognostic marker for various stages and settings of patients with NSCLC. Thus, we doubted the specificity of LIPI for ICI therapy. In this study, we aimed to investigate our hypothesis that LIPI is also a prognostic marker for NSCLC patients treated with cytotoxic chemotherapy. Unlike the previous study [1], we analyzed patients according to histological and genetic subsets.

\section{Materials and Methods}

We retrospectively collected three cohorts according to histological and genetic backgrounds: 1) Adenocarcinoma without active EGFR mutations; 2) NSCLC harboring active EGFR mutation, and 3) Squamous cell carcinoma. The patients met all the following criteria: 1) Between July 2007 and August 2017 at our hospital, first-line cytotoxic chemotherapy or EGFR-TKI monotherapy, irrespective of chemotherapeutic lines, being initiated for patients with wild-type EGFR adenocarcinoma and squamous cell carcinoma or for patients with mutant EGFR NSCLC, respectively; 2) Histologically or cytologically diagnosed with NSCLC; 3) For non-squamous NSCLC, the peptide nucleic acid-locked nucleic acid PCR clamp method or EGFR gene mutation analysis COBAS version 2 by LSI Medience Cooperation (Tokyo, Japan) confirmed wild-type or positive EGFR mutation status; 4) For adenocarcinoma with wild-type EGFR status, immunohistochemically negative or unknown anaplastic lymphoma kinase (ALK) rearrangement; 5) c-stage IIIB or IV based on the seventh tumor, node, metastasis (TNM) classification of lung cancer by the Union for International Cancer Control (UICC), or recurrence after curative-intent thoracic surgery or radiotherapy without adjuvant chemotherapy, and only for NSCLC with active EGFR mutation, c-stage IIIA was included when neither curative operation nor radiotherapy was intended; and 6) Available blood sample within 2 weeks prior to the first day of the first-line cytotoxic chemotherapy or EGFR-TKI monotherapy. We excluded patients who had received the first-line pembrolizumab, which Japanese medical insurance approved in December 2016. We collected clinical data from the electrical medical records, including age, sex, histology, EGFR mutation status, ALK rearrangement status, PD-L1 tumor proportion score, pretreatment laboratory data, chemotherapeutic regimens, its efficacy and survival. Pretreatment routine blood tests collected LDH level, total protein and albumin concentration, absolute counts of leucocyte and proportion of neutrophil in leukocyte. The dNLR was calculated by the following formula: neutrophil count/(leucocyte count - neutrophil count) [2]. The LIPI was formed by combination of $\mathrm{dNLR}>$ or $\leq 3$ and $\mathrm{LDH}>$ or $\leq$ ULN range of each center (225 IU/L in our hospital). Patients were categorized into three groups (good, no factors; intermediate, one factor; and poor, two factors) [1].

PFS and OS were defined as the interval from the first day of the regimen to the date of documented progressive disease (PD) or death, respectively. Based on Response Evaluation Criteria in Solid Tumors (RECIST) version 1.1 [9], we evaluated response to chemotherapy or EGFR-TKI. The data cut-off was July 31, 2018. The Osaka Police Hospital Ethics Committee approved this study. Considering the characteristic of anonymous and retrospective data, the written informed consents were waived in this study.

\section{Data analysis}

The continuous, categorical and survival data were expressed as the median with interquartile range (IQR), frequency, median with $95 \%$ confidential intervals (CIs), respectively. Using Fisher's exact test, Kruskal-Wallis rank sum test, Kaplan-Meier method and log-rank test, we compared the relative frequencies, continuous variables, survival times of three groups, respectively. Using the Bonferroni method for the multiple comparisons, $\mathrm{P}$ values were corrected. In multivariate Cox proportional hazard analyses, independent variables were selected and pre-defined according to the previous study [1]. The results were described as hazard ratios (HRs) with $95 \% \mathrm{CI}$. $\mathrm{P}<0.05$ was defined as statistically significant. All statistical analyses were performed with EZR (Saitama Medical Center, Jichi Medical University, Saitama, Japan), which is a graphical user interface for R (The R Foundation for Statistical Computing, Vienna, Austria) [10].

\section{Results}

We collected 175 patients with wild-type EGFR adenocarcinoma, 131 patients with NSCLC harboring active EGFR mutation and 110 patients with squamous cell carcinoma. These patients were divided into three groups by LIPI category. Tables 1-3 show patients' background, treatment, efficacy and laboratory data in each group. Table 4 shows outcomes of survival and treatment at the time of data cut-off.

In wild-type EGFR adenocarcinoma (good, intermediate and poor LIPI groups: median 19.6, 11.5 and 3.3 months, $\mathrm{P}<$ 0.01, respectively) and EGFR mutant NSCLC (45.4, 25.6 and 15.7 months, $\mathrm{P}<0.01$ ), the good LIPI group survived longer than the intermediate and poor LIPI groups (Fig. 1a, b). In contrast, in squamous cell carcinoma, there was no significant difference in OS among the three LIPI groups (13.7, 14.6 and 8.8 months, $\mathrm{P}=0.39$ ) (Fig. 1c). In EGFR mutant NSCLC, the PFS of good LIPI group was significantly longer than those of the other two groups (16.6, 12.6 and 8.3 months, $\mathrm{P}<0.01)$ (Fig. $2 \mathrm{~b}$ ), while no significant difference was found among the three groups of wild-type EGFR adenocarcinoma (6.3, 4.3 and 2.9 months, $\mathrm{P}=0.12$ ) and squamous cell carcinoma (4.9, 5.9 and 3.3 months, $\mathrm{P}=0.25$ ) (Fig. 2a, c).

As poorer prognostic factors of OS, multivariate Cox proportional hazard analyses found intermediate group (HR $1.49,95 \%$ CI $1.03-2.15, \mathrm{P}=0.04)$ of wild-type EGFR adenocarcinoma, and intermediate (HR 2.30, 95\% CI 1.33 - 3.99, $\mathrm{P}<0.01)$ and poor (HR 2.76, 95\% CI $1.03-7.42, \mathrm{P}=0.04)$ 


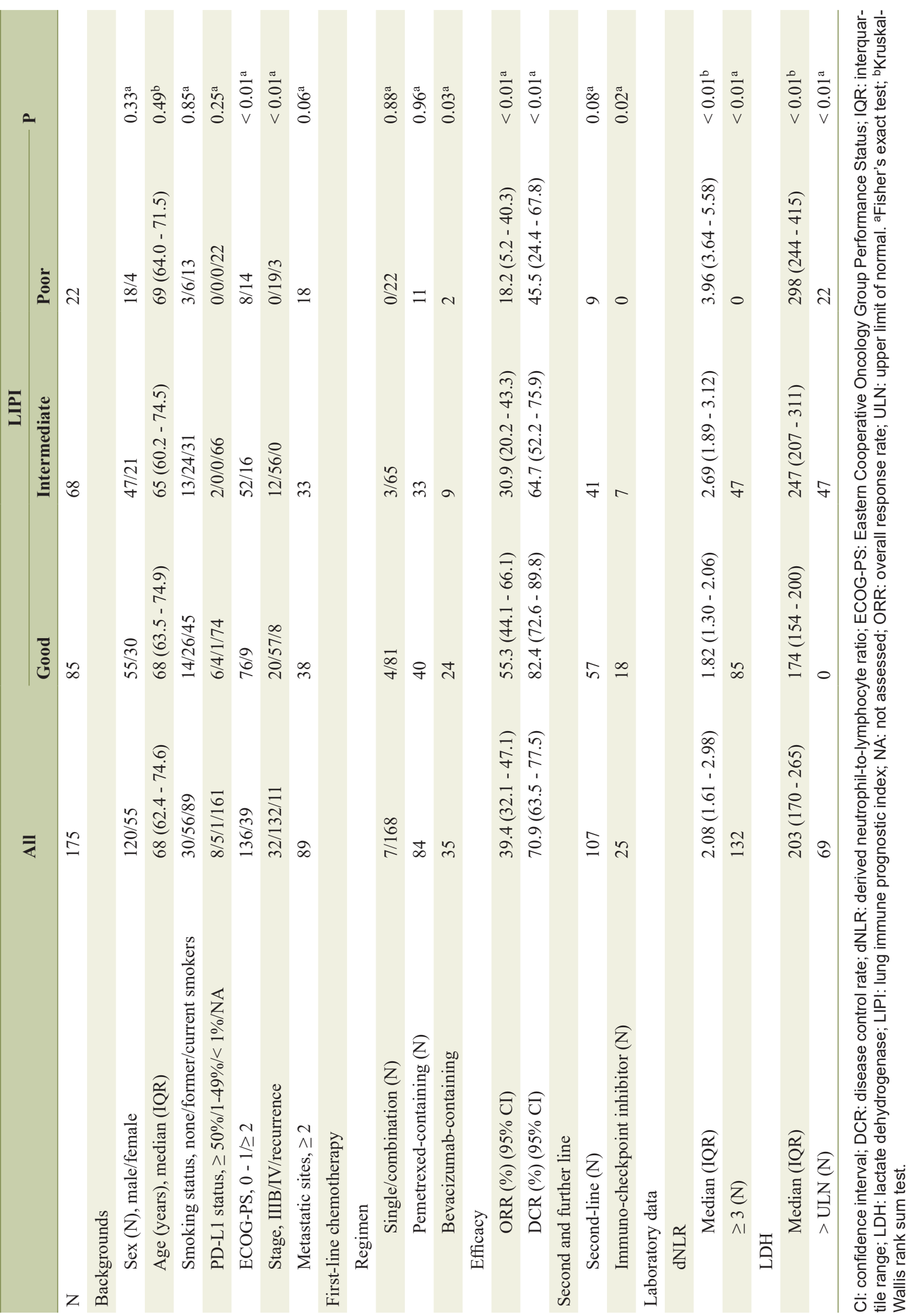




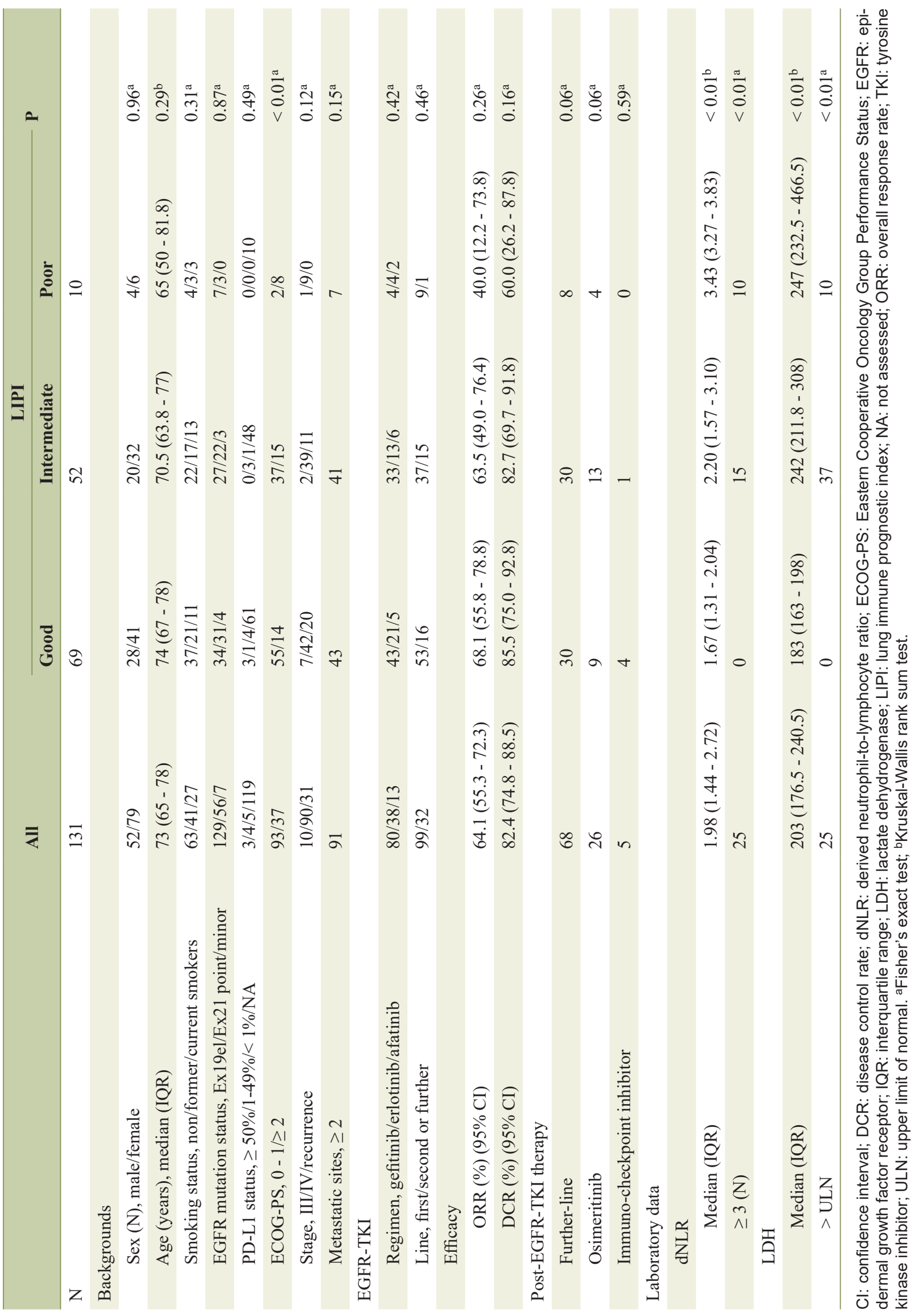




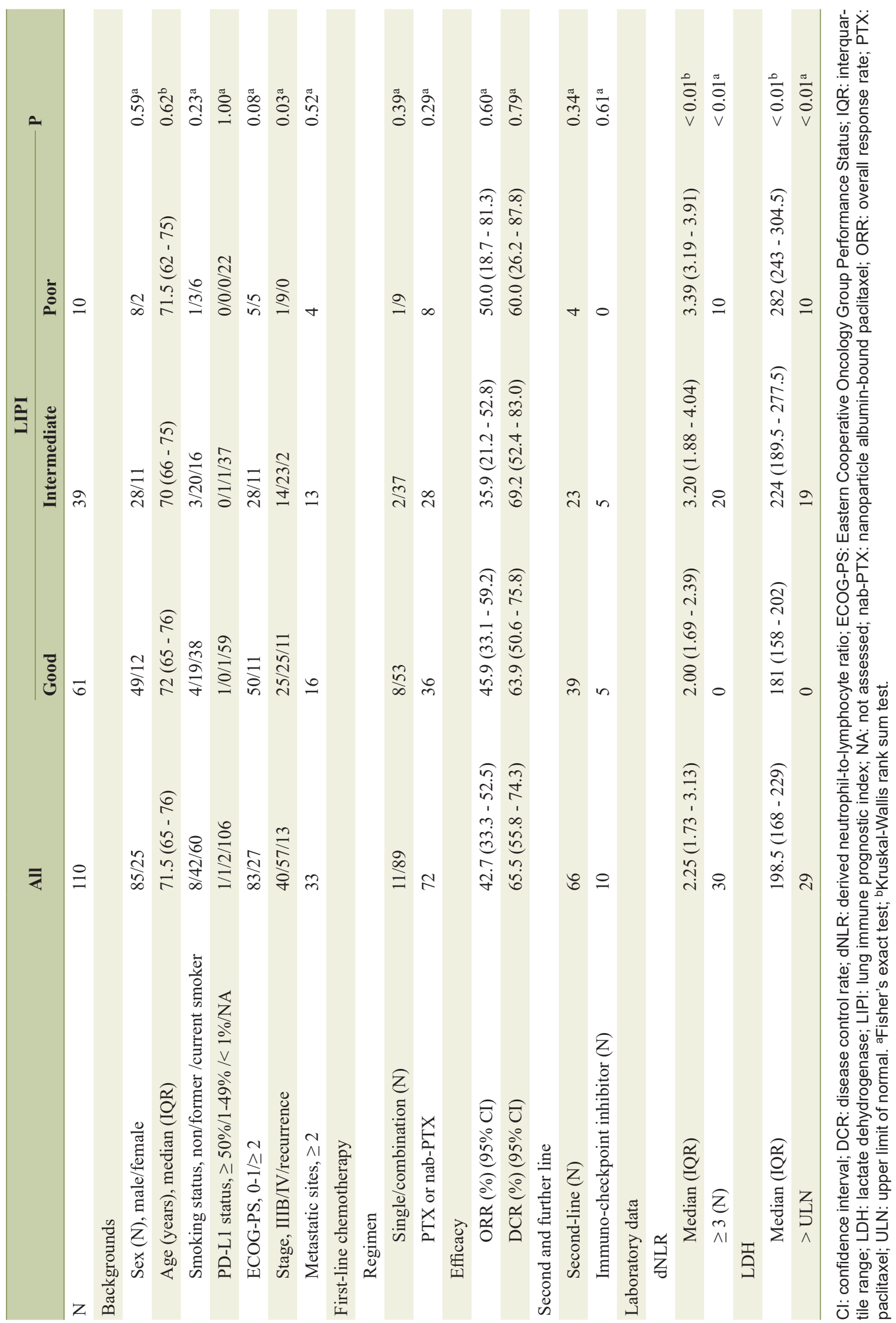




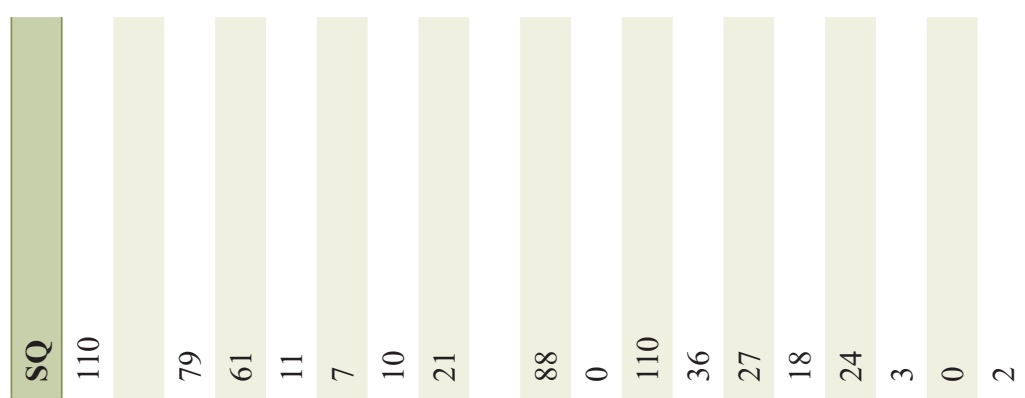

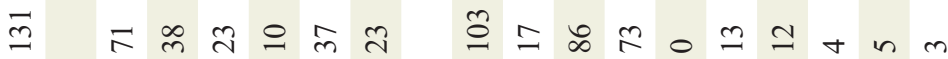

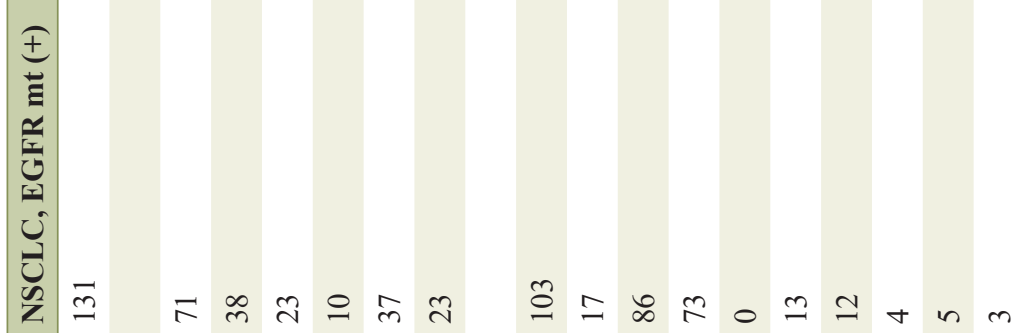

选

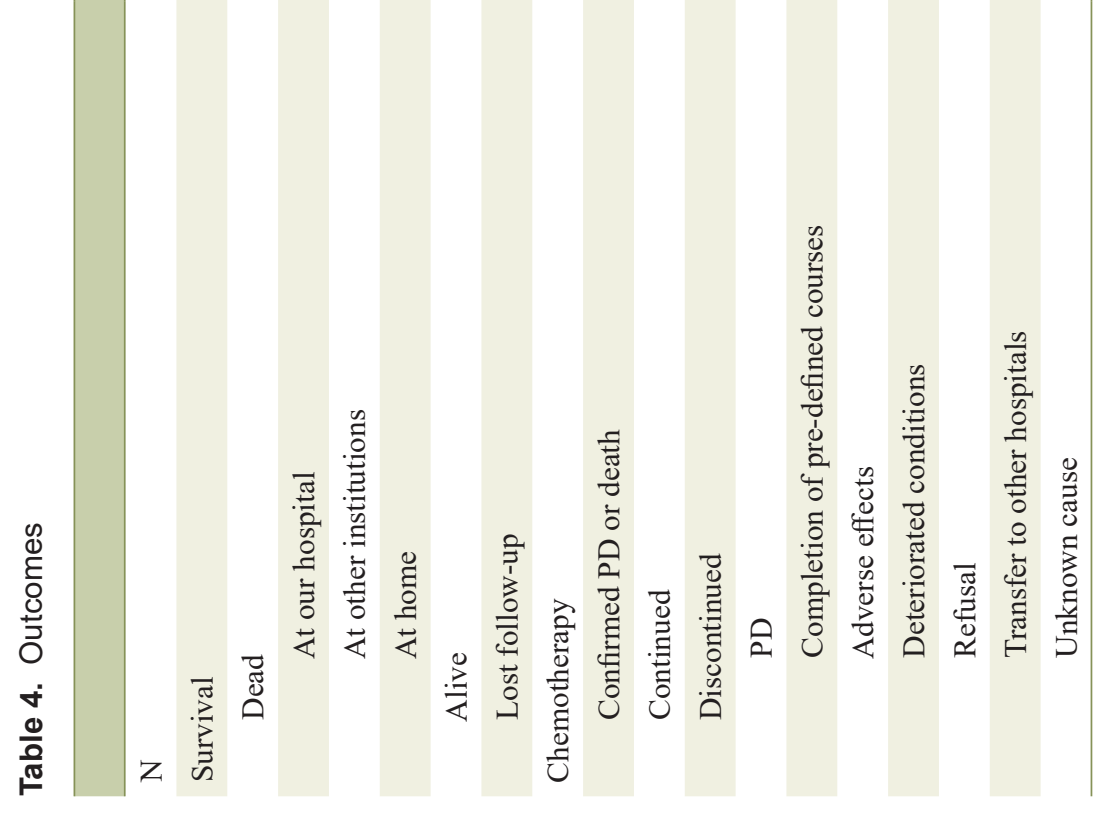



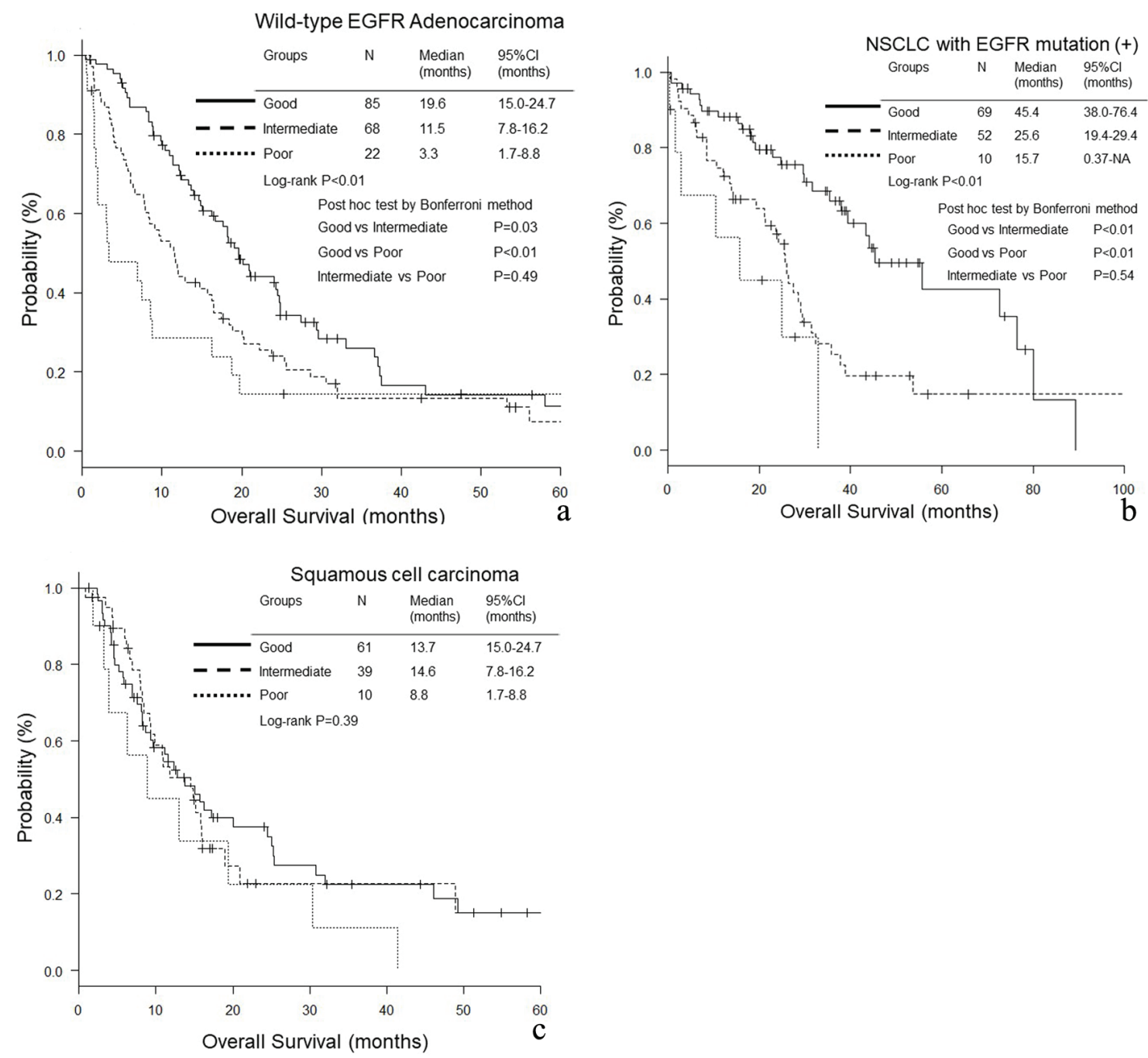

Figure 1. Overall survival (OS) according to lung immune prognostic index (LIPI) groups. (a) Adenocarcinoma without any driver mutations. (b) NSCLC with EGFR mutation. (c) Squamous cell carcinoma.

LIPI groups of EGFR mutant NSCLC (Table 5). Multivariate analyses detected intermediate (HR 1.57, 95\% CI $1.01-2.44$, $\mathrm{P}=0.04)$ and poor $(\mathrm{HR} 2.63,95 \% \mathrm{CI} 1.14-6.07, \mathrm{P}=0.02)$ LIPI groups as poor prognostic factors of PFS of EGFR mutant NSCLC (Table 6).

\section{Discussion}

This study demonstrated that pretreatment LIPI is a prognostic marker for adenocarcinoma with wild-type EGFR and for NSCLC with active EGFR mutation, but not for squamous cell carcinoma. LIPI showed different trends as a prognostic marker according to histological and genetic subsets.

Our multivariate analyses and comparisons of OS and PFS showed this marker as independent prognostic factors of OS of first-line cytotoxic chemotherapy for adenocarcinoma with wild-type EGFR, and of OS and PFS of EGFR-TKI therapy for
NSCLC with active EGFR mutation. Our results were contrary to the previous study, in which LIPI was significantly correlated with outcomes for ICI, but not for chemotherapy [1]. In the study by Mezquita, chemotherapy cohort $(\mathrm{N}=162)$ was composed only of patients who had received second or later chemotherapy, and included various subsets of NSCLC: $72 \%$ of adenocarcinoma, $14 \%$ of squamous cell carcinoma, $23 \%$ of positive EGFR mutation or ALK rearrangement [1]. Our study investigated first-line chemotherapy or first EGFR-TKI therapy, and divided patients by subsets. Thus, LIPI is not only a specific marker for ICI therapy, but also is a useful prognostic marker for some specific subsets of NSCLC.

In our study, LIPI was not an independent prognostic factor of chemotherapy for squamous cell carcinoma. There was a different trend of LIPI as a prognostic factor between squamous cell carcinoma and other subsets. The difference of hematological markers according to subsets was observed in our series of previous studies. As a prognostic factor of OS, 

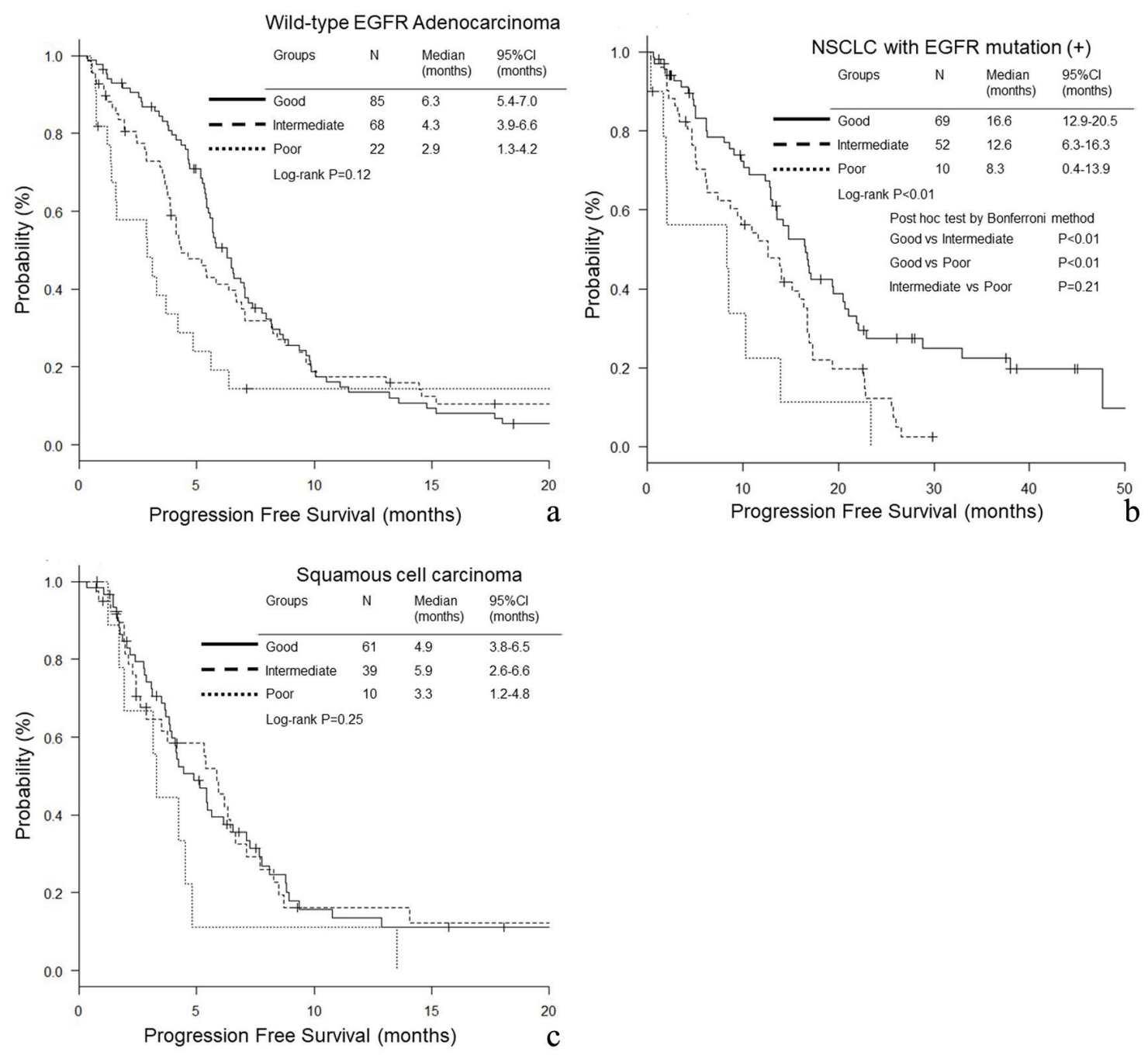

Figure 2. Progression-free survival (PFS) according to lung immune prognostic index (LIPI) groups. (a) Adenocarcinoma without any driver mutations. (b) NSCLC with EGFR mutation. (c) Squamous cell carcinoma.

neither NLR nor modified Glasgow prognostic score (mGPS) was useful for squamous cell carcinoma [11], while mGPS, but not NLR, was selected for adenocarcinoma with wild-type EGFR [12]. On the other hand, NLR was significantly useful for NSCLC harboring mutant EGFR, though mGPS was not investigated in our previous study [13]. Little has been reported on mGPS as a prognostic factor for patients with driver mutations. Thus, hematological biomarkers variously responded among subsets of NSCLC. It may be necessary to evaluate biomarkers by histological and genetic subsets.

Our study had some limitations. First, we are afraid of case bias owing to our retrospective, single-centered and small sample-sized study. Second, in terms of recent fast advance in NSCLC treatment, the regimens that our patients received are becoming outdated. Our study included many patients who had initiated their treatment and become unable to proceed to the further lines until the approval of ICIs or third-generation EGFR-TKI. The new era of next generation treatment is com- ing, and it needs more cases to confirm LIPI as a useful marker of advanced NSCLC.

\section{Conclusions}

LIPI was an independent prognostic factor of chemotherapy for adenocarcinoma with wild-type EGFR and of EGFR-TKI for NSCLC harboring mutant EGFR. Thus, LIPI was not a specific biomarker for ICI therapy, but a useful biomarker for chemotherapy and EGFR-TKI therapy in specific subsets of NSCLC. The optimal prognostic markers may be different among subsets of NSCLC.

\section{Acknowledgments}

We are grateful to Tsunehiro Tanaka, Kanako Nishimatsu, 


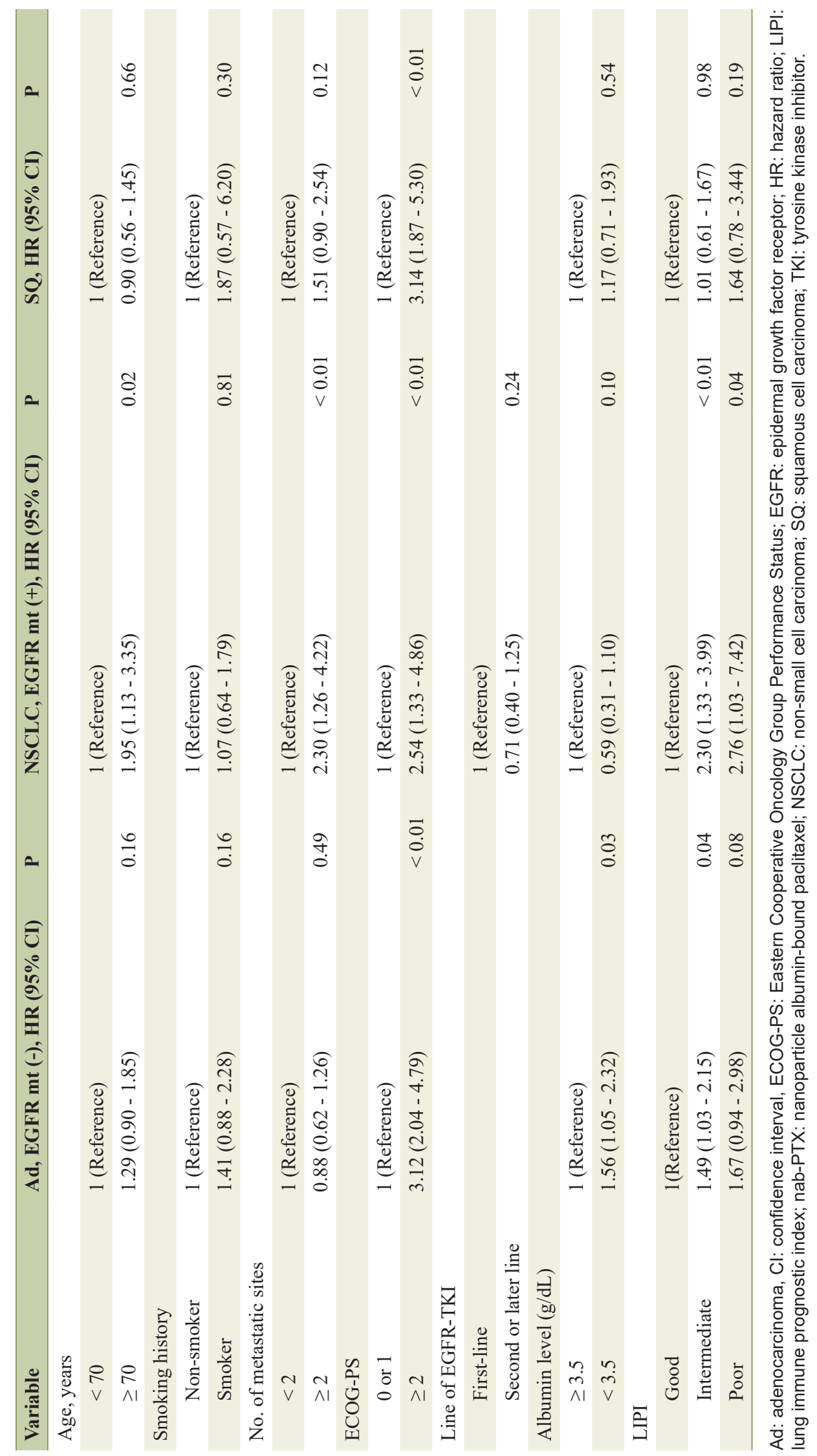



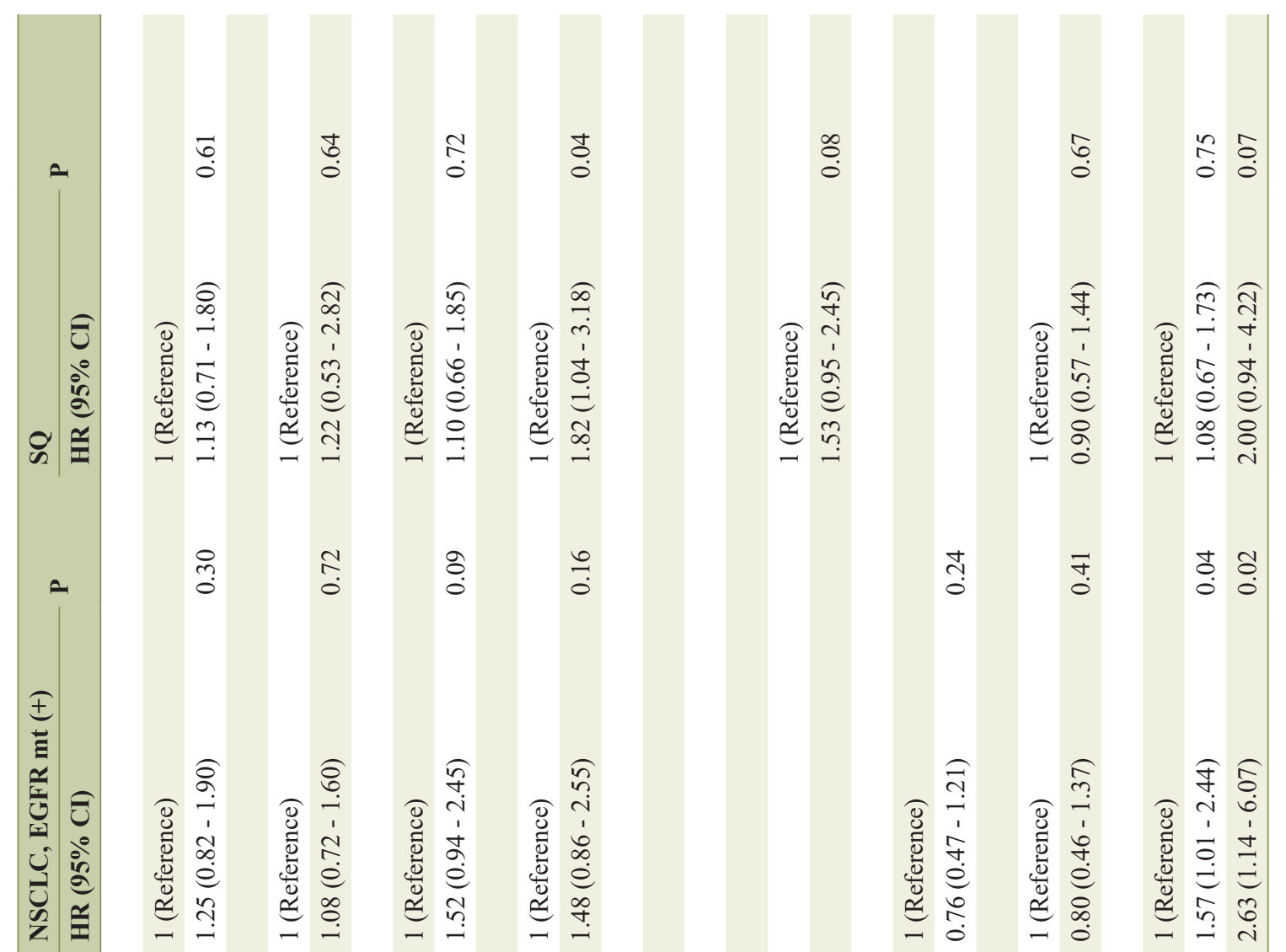

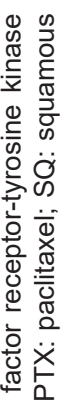

흠

हैं

कั

है

음

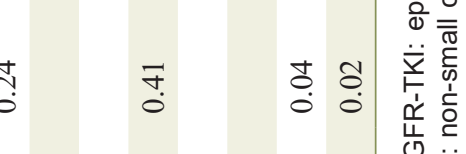

记

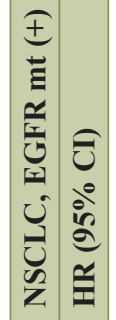

$-\rightarrow-$ -

$-\dot{-}$

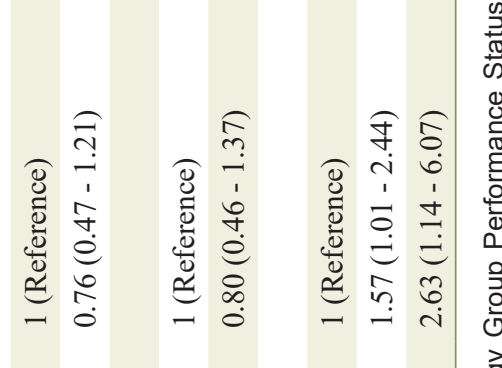

这

है

는 은

응 운

일

ते

응 은

- $\begin{array}{lllll}0 & 0 & 0 & 0 & 0 \\ 0 & 0 & 0 & 0\end{array}$

\&

$\sum_{\frac{1}{\pi}}^{\infty} \stackrel{0}{\frac{0}{\pi}}$

产芒
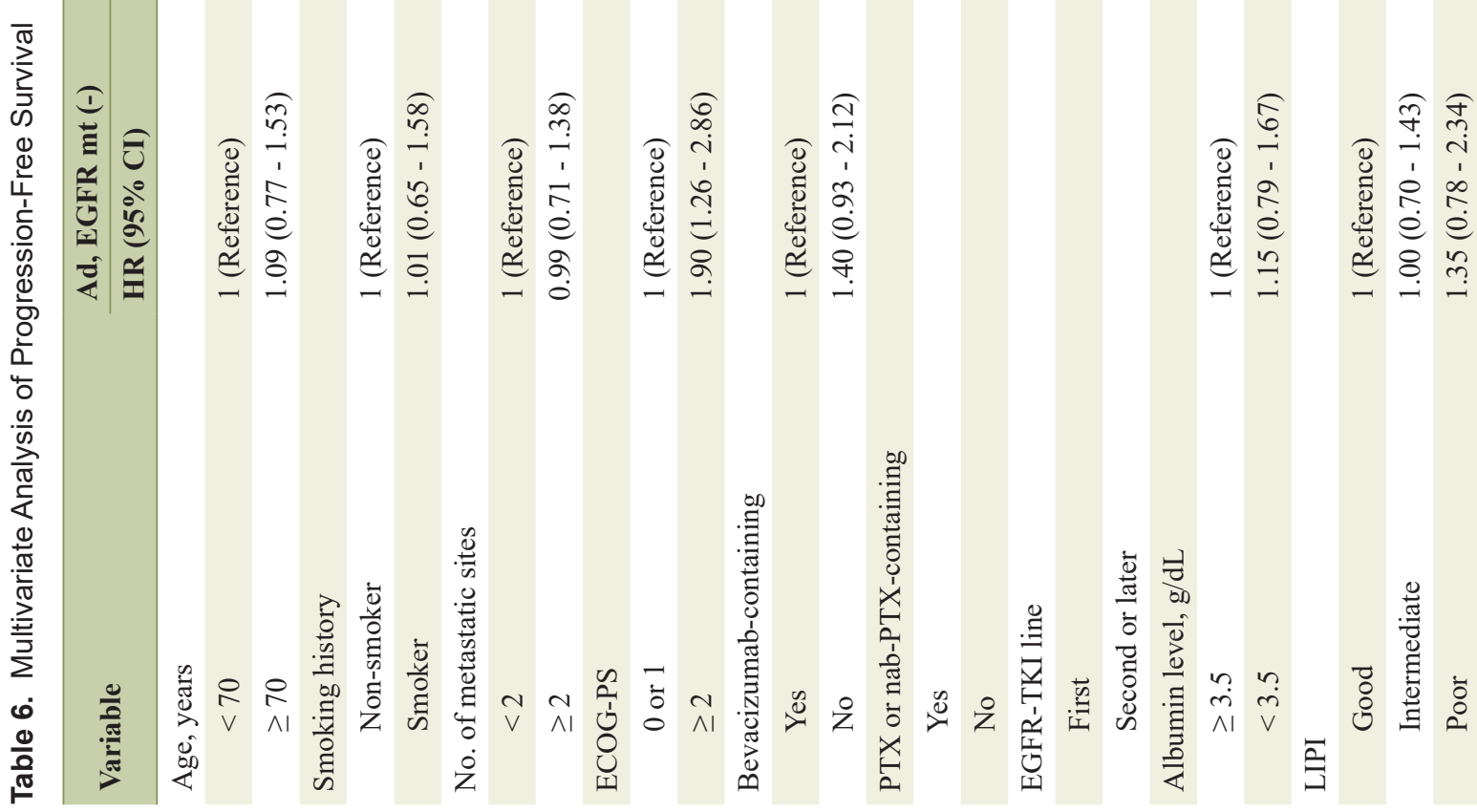

동

离

is

ò क

品음

त्ञ

离

옹

送

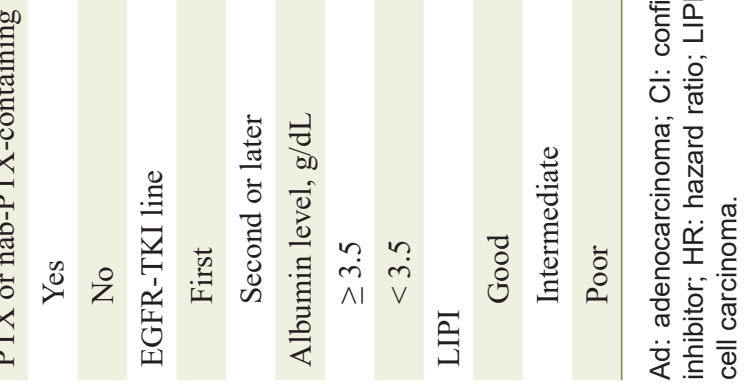

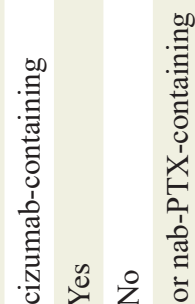


Shouko Ikuta, Saori Ikebe, Hideyasu Okada and Kazuki Hashimoto at the Department of Respiratory Medicine, Osaka Police Hospital for their detailed medical records, diagnosis, treatment and care of their patients.

\section{Conflict of Interest}

The authors declare that they have no conflict of interest.

\section{Grant Support}

None.

\section{References}

1. Mezquita L, Auclin E, Ferrara R, Charrier M, Remon J, Planchard D, Ponce S, et al. Association of the lung immune prognostic index with immune checkpoint inhibitor outcomes in patients with advanced non-small cell lung cancer. JAMA Oncol. 2018;4(3):351-357.

2. Proctor MJ, McMillan DC, Morrison DS, Fletcher CD, Horgan PG, Clarke SJ. A derived neutrophil to lymphocyte ratio predicts survival in patients with cancer. Br J Cancer. 2012;107(4):695-699.

3. Danner BC, Didilis VN, Wiemeyer S, Stojanovic T, Kitz $\mathrm{J}$, Emmert A, Fuzesi L, et al. Long-term survival is linked to serum LDH and partly to tumour LDH-5 in NSCLC. Anticancer Res. 2010;30(4):1347-1351.

4. Lee DS, Park KR, Kim SJ, Chung MJ, Lee YH, Chang JH, Kang JH, et al. Serum lactate dehydrogenase levels at presentation in stage IV non-small cell lung cancer: predictive value of metastases and relation to survival outcomes. Tumour Biol. 2016;37(1):619-625.
5. Zhu L, Li X, Shen Y, Cao Y, Fang X, Chen J, Yuan Y. A new prognostic score based on the systemic inflammatory response in patients with inoperable non-small-cell lung cancer. Onco Targets Ther. 2016;9:4879-4886.

6. Gu XB, Tian T, Tian XJ, Zhang XJ. Prognostic significance of neutrophil-to-lymphocyte ratio in non-small cell lung cancer: a meta-analysis. Sci Rep. 2015;5:12493.

7. Peng B, Wang YH, Liu YM, Ma LX. Prognostic significance of the neutrophil to lymphocyte ratio in patients with non-small cell lung cancer: a systemic review and meta-analysis. Int J Clin Exp Med. 2015;8(3):3098-3106.

8. Yin Y, Wang J, Wang X, Gu L, Pei H, Kuai S, Zhang Y, et al. Prognostic value of the neutrophil to lymphocyte ratio in lung cancer: A meta-analysis. Clinics (Sao Paulo). 2015;70(7):524-530.

9. Eisenhauer EA, Therasse P, Bogaerts J, Schwartz LH, Sargent D, Ford R, Dancey J, et al. New response evaluation criteria in solid tumours: revised RECIST guideline (version 1.1). Eur J Cancer. 2009;45(2):228-247.

10. Kanda Y. Investigation of the freely available easy-touse software 'EZR' for medical statistics. Bone Marrow Transplant. 2013;48(3):452-458.

11. Minami S, Ihara S, Komuta K. Pretreatment lymphocyte to monocyte ratio as a prognostic marker for advanced pulmonary squamous cell carcinoma treated with chemotherapy. J Clin Med Res. 2018;10(8):657-664.

12. Minami S, Ihara S, Kim SH, Yamamoto S, Komuta K. Lymphocyte to monocyte ratio and modified Glasgow prognostic score predict prognosis of lung adenocarcinoma without driver mutation. World J Oncol. 2018;9(1):1320.

13. Minami S, Ogata Y, Ihara S, Yamamoto S, Komuta K. Neutrophil-to-lymphocyte ratio predicts overall survival of advanced non-small cell lung cancer harboring mutant epidermal growth factor receptor. World J Oncol. 2017;8(6):180-187. 